\title{
FISH detection of trisomy 21 in interphase by the simultaneous use of two differentially labelled cosmid contigs
}

\author{
A F Davies, L Barber, $M$ Murer-Orlando, $M$ Bobrow, M Adinolfi
}

\begin{abstract}
Techniques have been reported in which fluorescence in situ hybridisation (FISH) and cosmid probes are used to detect trisomy 21 (and other abnormalities involving chromosomes $X, Y, 13$, and 18) on uncultured amniocytes. However the detection rate of trisomy 21 is lower than for the other anomalies owing to a larger number of uninformative results and false negatives.

We report the simultaneous use of two differentially labelled cosmid contigs to improve the detection rate of trisomy 21 on uncultured amniocyte samples thus allowing the prenatal diagnosis of Down's syndrome even if only few labelled nuclei are available.
\end{abstract}

( $\mathcal{F}$ Med Genet 1994;31:679-685)

A reliable test for trisomy 21 on uncultured prenatal material would obviate the need for cell culture, thus saving resources and allowing diagnoses on cells from sources not amenable to culture, for example, fetal cells sorted from maternal blood ${ }^{12}$ and transcervical flushing. ${ }^{34}$

One requirement for successful interphase screening of numerical chromosomal aneuploides by non-isotopic in situ hybridisation (NISH) is the availability of reliable chromosome specific probes which produce strong, discrete signals in uncultured cell preparations. Chromosome specific repetitive probes such as those derived from $\alpha$ satellite regions produce large discrete signals in both metaphase and interphase nuclei; these are visible even in the presence of undesirable background staining which is often encountered in preparations of uncultured cells. Specific repetitive probes are available for chromosomes $\mathrm{X}, \mathrm{Y}$, and 18; however, owing to the high degree of sequence homology between the repetitive regions of chromosomes 21 and 13, no chromosome-specific repeat probe exists for either. The plasmid probe $\mathrm{L} 1.26^{5}$ specific for the centromeres of chromosomes 21 and 13 has been used in interphase with unsatisfactory results. ${ }^{67}$ This region of chromosome 21 is highly polymorphic, leading to variations in signals size; in extreme cases signals produced on chromosome 21 by the L1.26 probe may be barely visible in interphase. Secondly the acrocentric chromosomes, including 21 and 13, associate by their centromeres at the nucleolus during interphase, possibly resulting in coalescence of signals in some cells.
In 1986 Julien et al reported the first prenatal diagnosis of trisomy 21 on uncultured amniocytes, using restriction digested DNA obtained from flow sorted chromosomes 21. However, subsequent investigations with chromosome 21 derived libraries (that is, pooled probes constituting the whole of chromosome 21) produced unsatisfactory results when applied to nuclei in interphase owing to the large size and consequent coalescence of signals. ${ }^{9-12}$ The frequency of nuclei with three signals in cultured amniotic cells from fetuses with trisomy 21 varied from 10 to $52 \% .{ }^{11}$

A number of groups have investigated the use of pools of overlapping cosmids (cosmid contigs) for detection of numerical chromosomal aneuploidies. Klinger et $a l,{ }^{13}$ Ried et $a l,{ }^{14}$ and Zheng $e t a^{12}$ compared the results of analysing amniotic cells by conventional cytogenetic techniques and FISH tests on nuclei in interphase, using probes derived from subregions of human chromosomes 13, 18, 21, X, and $\mathrm{Y}$. These investigations were carried out on samples which had already been tested by conventional cytogenetic techniques. The overall detection efficiency was less on uncultured amniocytes than on cultured peripheral blood lymphocytes and amniocytes, and the hybridisation efficiency was on the whole lower in trisomic samples.

In the first prospective clinical project, which used FISH for the detection of chromosome aneuploidies, Ward et $a l^{15}$ tested 4500 uncultured amniotic samples with $\mathrm{X}, \mathrm{Y}, 13,18$, and 21 specific probes. A sample was considered to be aneuploid when $70 \%$ or more hybridised nuclei displayed the same hybridisation pattern for a specific probe. The overall detection rate for aneuploidies was $73.3 \%$; that for trisomy 21 was lower $(63.3 \%)$ since of 60 samples tested 17 yielded uninformative results and five cases were false negatives.

These results suggest a problem inherent in trisomy 21 detection on uncultured amniocytes. We have observed the presence of spurious signals on uncultured amniocytes following FISH; these presumably result from hybridisation of the probe to low affinity sites or nonspecific binding of fluorochrome labelled ligand to the cell cytoskeleton. Whenever these spurious signals are counted as true signals in a normal or trisomic sample the statistical value of the results is diminished. We report the results of comparing several chromosome 21 derived probes for the detection of numerical variations of chromosome 21 and finally describe an improvement in the detection of trisomy 21 on uncultured amniocytes by the sim- 
ultaneous use of two differentially labelled cosmid contigs.

We used two contig probes mapping to 21 22 which contains the region involved in Down's syndrome, and owing to its location away from the centromere should allow the detection of trisomy resulting from Robertsonian translocation, although this aspect is not investigated in this study. Signals were only accepted and counted if they were composed of a doublet of one red and one green signal. Using this technique three doublet signals were counted in at least $50 \%$ of the trisomy 21 uncultured amniocytes; this pattern was not seen or was seen in only a low number $(<5 \%)$ of nuclei from normal amniocytes.

\section{Materials and methods}

The various chromosome 21 derived probes were tested initially on metaphase and interphase preparations from phytohaemagglutinin (PHA) stimulated peripheral blood lymphocytes. They were then used on 59 uncultured amniotic samples, of which nine were from fetuses with trisomy 21 , and 25 samples of cultured amniotic cells.

\section{INTERPHASE PREPARATIONS}

Slides of uncultured amniotic cells were prepared from $\sim 2 \mathrm{ml}$ of amniotic fluid. They were washed once with phosphate buffered saline (PBS) and then treated with $0.05 \mathrm{~mol} / 1 \mathrm{KCl}$ at $37^{\circ} \mathrm{C}$ for 20 minutes. The cells were then fixed using three successive incubations in Carnoy's solution (3:1 methanol:acetic acid). Suspended in this fixative, the cells were stored at $-20^{\circ} \mathrm{C}$ for periods from one day to one year before being analysed.

One day before FISH testing, the cells were centrifuged, resuspended in approximately $100 \mu$ l Carnoy's solution, and used to prepare two slides. The slides were refixed and then incubated at $42^{\circ} \mathrm{C}$ overnight on a hotplate.

Venous blood samples were collected in heparin and the lymphocytes, isolated with Histopaque 1083 (Sigma Chemical Co, UK), were first lysed with $0.075 \mathrm{~mol} / 1 \mathrm{KCl}$ and then fixed, in suspension, with three changes of Carnoy's solution. At this stage they were stored at $-20^{\circ} \mathrm{C}$ for up to one month. One day before FISH, the nuclei were dropped onto methanol washed slides, refixed, and stored at $4^{\circ} \mathrm{C}$ overnight.

FISH ON CULTURED SAMPLES

Amniotic fluid cells were cultured by standard cytological techniques and harvested by trypsinisation. ${ }^{16}$ They were then lysed by suspension in $0.05 \mathrm{~mol} / 1$ potassium chloride at $37^{\circ} \mathrm{C}$ for 20 minutes and fixed by three successive incubations in Carnoy's solution and stored at $-20^{\circ} \mathrm{C}$ for periods from one day to one year.

Metaphase chromosomes from peripheral blood lymphocytes were prepared after stimulation of the cells with phytohaemagglutinin (PHA) and synchronisation with thymidine. ${ }^{17}$ After treatment with $0.075 \mathrm{~mol} / 1 \mathrm{KCl}$ the cells were fixed with three changes of Carnoy's solution and stored at $-20^{\circ} \mathrm{C}$ until use. One day before FISH they were dropped onto methanol washed slides and stored at $4^{\circ} \mathrm{C}$ overnight.

PROBES

The probes used in this investigation are listed in table 1. Plasmid and cosmid sequences, labelled by nick translation with biotin-14dATP or digoxigenin-11-dUTP (Bio-Nick Labeling System or Nick Translation System respectively, BRL Life Technologies, USA), were purified using Sephadex G-50 spin columns. $^{18}$

The YAC clone yGART2 (kindly provided by Andreas Gnirke $^{19}$ ) was prepared in agarose plugs $^{2021}$ and the whole yeast DNA, including the YAC, was purified by $\beta$ agarose digestion and successive extractions with phenol and chloroform. The total yeast DNA was then labelled with biotin-14-dATP as above.

Seventeen cosmid clones were obtained from the chromosome 21 library number 102 (L4/ FS21) at the ICRF Reference Library Database; they had been selected after screening on request with one of three probes (D21S55,

Table 1 Details and source of probes used in this study

\begin{tabular}{llll}
\hline Name & Type & Site of specific hybridisation & Source of clone \\
\hline L1.26 & Plasmid & 21 centromere \& 13 centromere & Devilee et al \\
ICRFc102D12118 & Cosmid & $21 \mathrm{q} 22.3$ & ICRF RLDB \\
yGART2 & YAC & $21 \mathrm{q} 22.1$ & A Gnirke \\
ICRFc102A0779 & Cosmid & $21 \mathrm{q} 22.3$ & ICRF RLDB \\
ICRFc102B02105 & Cosmid & $21 \mathrm{q} 22.3$ & ICRF RLDB \\
ICRFc102C07132 & Cosmid & $21 \mathrm{q} 22.3$ & ICRF RLDB \\
ICRFc102A07120 & Cosmid & $21 \mathrm{q} 22.3$ & ICRF RLDB \\
ICRFc102D06126 & Cosmid & $21 \mathrm{q} 22.3$ & ICRF RLDB \\
ICRFc102E03129 & Cosmid & $21 \mathrm{q} 22.3$ & ICRF RLDB \\
ICRFc102C10134 & Cosmid & $21 \mathrm{q} 22.3$ & ICRF RLDB \\
ICRFc102C0639 & Cosmid & $21 \mathrm{q} 22.3$ & ICRF RLDB \\
ICRFc102F0458 & Cosmid & $21 \mathrm{q} 22.3$ & ICRF RLDB \\
ICRFc102G0563 & Cosmid & $21 \mathrm{q} 22.3$ & ICRF RLDB \\
ICRFc102E0275 & Cosmid & $21 \mathrm{q} 22.3$ & ICRF RLDB \\
ICRFc102H01108 & Cosmid & $21 \mathrm{q} 22.3$ & ICRF RLDB \\
ICRFc102F01129 & Cosmid & $21 \mathrm{q} 22.3$ & ICRF RLDB \\
ICRFc102F10130 & Cosmid & $21 \mathrm{q} 22.3$ & ICRF RLDB \\
ICRFc102A1140 & Cosmid & $21 \mathrm{q} 22.3$ & ICRF RLDB \\
ICRFc102H0763 & Cosmid & $21 \mathrm{q} 22.3$ & ICRF RLDB \\
ICRFc102C0288 & Cosmid & $21 \mathrm{q} 22.3$ & ICRF RLDB \\
cCMP21.a & Cosmid contig & $21 \mathrm{q} 22$ proximal & Y L Zheng
\end{tabular}

ICRF RLDB: Reference Library Database, Imperial Cancer Research Fund, Lincoln's Inn Fields, London WC2A 3PX.

A Gnirke: this clone was a generous gift from Andreas Gnirke, Washington University School of Medicine, St Louis, USA. Y L Zheng: this cosmid contig was a kind gift from Y L Zheng and N Carter, University of Cambridge, UK. 
D12S3, or F33B4) mapping to the 21q22.3 region. ${ }^{22}$ Each cosmid was tested by FISH on lymphocyte metaphase spreads, by fingerprint analysis using $\operatorname{Hinf}^{2324}$ and restriction digestion using EcoRI and BamHI. As a result of this a pool of three overlapping cosmids (ICRFc102E0275; ICRFc102HO1108; ICRFc102F01129), which covered approximately $80 \mathrm{~kb}$, was selected for further investigation on nuclei in interphase. This contig will be referred to as $242 \mathrm{c}$.

The cosmid contig cCMP21.a (labelled with biotin) was kindly provided by Yun Ling Zheng and Nigel Carter (University of Cambridge).

\section{IN SITU HYBRIDISATION}

All slides were treated with $100 \mu \mathrm{g} / \mathrm{ml}$ RNAse A (Sigma Chemical Co) in $2 \times \mathrm{SSC}$ at $37^{\circ} \mathrm{C}$ for one hour, washed once in $2 \times \mathrm{SSC}$, once in PBS, and then dehydrated in an ethanol series $(70 \%, 90 \%, 100 \%)$. The following optional steps were assessed with amniocyte slides before dehydration in an attempt to increase the percentage of informative nuclei, preserve target morphology, and decrease loss of material during processing. These included treatment with $10 \mu \mathrm{g} / \mathrm{ml}$ pepsin in $10 \mathrm{mmol} / 1 \mathrm{HCl}$ at $37^{\circ} \mathrm{C}$ for 10 minutes or $10 \mathrm{ng} / \mathrm{ml}$ proteinase $\mathrm{K}$ in $20 \mathrm{mmol} / 1 \mathrm{Tris}-\mathrm{HCl}, 2 \mathrm{mmol} / 1 \mathrm{CaCl}_{2}, \mathrm{pH} 7.5$ at $37^{\circ} \mathrm{C}$ for 10 minutes, followed by additional fixation in $1 \%$ paraformaldehyde in PBS, $1 \%$ $\mathrm{MgCl}_{2}$ for 10 minutes.

Repetitive probes, at $2 \mathrm{ng} / \mu \mathrm{l}$ in $50 \%$ formamide, $10 \%$ dextran sulphate, $2 \times \mathrm{SSC}$, $40 \mathrm{mmol} / 1 \quad \mathrm{NaH}_{2} \mathrm{PO}_{4}, \quad 500 \mu \mathrm{g} / \mathrm{ml} \quad$ ssDNA (sheared sonicated salmon sperm DNA), pH 7, were denatured by heating to $75^{\circ} \mathrm{C}$ for three minutes and were then cooled rapidly on ice ready for application to the slide.

Specific sequence probes were prehybridised with CotI DNA (BRL Life Technologies) present at 10 to 50 fold excess by suspending the DNA probe at $7 \mathrm{ng} / \mu \mathrm{l}$ (cosmids) or $3.5 \mathrm{ng} / \mu \mathrm{l}$ (YACs) together with the $\operatorname{Cot} I$ DNA in a solution of $50 \%$ formamide, $10 \%$ dextran sulphate, $2 \times$ SSC, pH 7, containing ssDNA at 100 fold excess. The DNA was denatured by heating to $75^{\circ} \mathrm{C}$ for three minutes and incubated at $37^{\circ} \mathrm{C}$ for one or three hours.

Immediately before application of the probe, the chromosomal DNA was denatured by incubation of the slides in $70 \%$ formamide, $2 \times \mathrm{SSC}, 40 \mathrm{mmol} / 1 \mathrm{NaH}_{2} \mathrm{PO}_{4}, \mathrm{pH} 7$, for $2 \frac{1}{2}$ minutes at $65^{\circ} \mathrm{C}$. After being dehydrated by washing in a $4^{\circ} \mathrm{C}$ ethanol series $(70 \%, 90 \%$, $100 \%$ ethanol), the slides were air dried.

The denatured probes were applied to the slides under coverslips which were sealed with rubber solution glue and incubated at $37^{\circ} \mathrm{C}$ for approximately 16 hours.

After hybridisation, the slides were washed three times (five minutes per wash) in $50 \%$ formamide, $2 \times \mathrm{SSC}, \mathrm{pH} 7$ at $45^{\circ} \mathrm{C}$, three times (five minutes per wash) in $0.1 \times \mathrm{SSC}, \mathrm{pH} 7$ at $60^{\circ} \mathrm{C}$, and once for five minutes in $4 \times \mathrm{SSC}$, $0.05 \%$ Tween-20, pH 7. They were then blocked with normal rabbit serum diluted $1 / 10$ in $4 \% \mathrm{BSA}, 4 \times \mathrm{SSC}, \mathrm{pH} 7$.
After hybridisation with biotin labelled probes, the slides were incubated with avidinFITC (Vector Laboratories, USA) $(5 \mu \mathrm{g} / \mathrm{ml}$ in $0.1 \% \mathrm{BSA}, 4 \times \mathrm{SSC}, \mathrm{pH} 7$ ) for 20 minutes followed by three washes (five minutes each) in $4 \times$ SSC, $0.05 \%$ Tween-20, pH 7 . They were then incubated for 20 minutes in biotinylated anti-avidin (Vector Laboratories, USA) (diluted to $5 \mu \mathrm{g} / \mathrm{ml}$ as above), washed three times, and finally incubated again in avidinFITC. After one wash in $4 \times \mathrm{SSC}, 0.05 \%$ Tween-20, pH 7, and two washes in PBS, the slides were mounted in Vectashield antifading medium (Vector Laboratories, USA) containing $0.3 \mu \mathrm{g} / \mathrm{ml}$ propidium iodide as counterstain. Results were visualised under a Zeiss Axioplan microscope using the Zeiss double band pass filter set 23, or filter 5 of the Pinkel Filter Wheel No 1 supplied with a cooled CCD camera and image analysis system (Digital Scientific, UK).

Signals produced using digoxigenin labelled probes were developed by incubating the slides in TRITC-sheep anti-digoxigenin (Boehringer Mannheim GmbH, Germany) $(10 \mu \mathrm{g} / \mathrm{ml}$ in $0 \cdot 1 \% \mathrm{BSA}, 4 \times \mathrm{SSC}, \mathrm{pH} 7$ ) for 20 minutes. The slides were then washed once in $4 \times \mathrm{SSC}$, $0.05 \%$ Tween-20, pH 7, twice in PBS and mounted in Vectashield containing $0.1 \mu \mathrm{g} / \mathrm{ml}$, 4',6-diamidino-2-phenylindole (DAPI) as counterstain. Visualisation was achieved as above using Zeiss filter sets 02 and 23 for DAPI and TRITC respectively, or filters 2 and 3 respectively of the Pinkel Filter Wheel No 1 as described above.

When biotin and digoxigenin labelled probes were used simultaneously, signals were developed by incubating the slides in TRITCsheep anti-digoxigenin and avidin-FITC (10 and $5 \mu \mathrm{g} / \mathrm{ml}$ respectively in $0.1 \%$ BSA, $4 \times$ SSC, $\mathrm{pH} 7$ ) for 20 minutes followed by three washes (five minutes each) in $4 \times \mathrm{SSC}$, $0.05 \%$ Tween-20, $\mathrm{pH}$. They were then treated once with biotinylated anti-avidin and once more with avidin-FITC as above and mounted in Vectashield antifading medium containing $0 \cdot 1 \mu \mathrm{g} / \mathrm{ml}$ DAPI. Slides were visualised as above using Zeiss filters 02 and 23 for DAPI and the hybridisation signals respectively, or filters 2 and 5 respectively of Pinkel Filter Wheel No 1.

All analyses were performed by direct visualisation down the microscope; the CCD camera and image analysis system were used for photographic purposes only.

\section{SCORING OF RESULTS}

Nuclei were scored as having $0,1,2,3,4$, or $>4$ hybridisation signals, those showing one or more being described as "hybridised nuclei". Owing to the low percentage of hybridised nuclei in amniocyte samples, these data were expressed as a percentage of hybridised nuclei showing $1,2,3,4$, or $>4$ signals.

Fifty hybridised nuclei were counted per sample when possible.

Those samples with 10 or fewer hybridised nuclei were considered uninformative. If a sample appeared trisomic up to 100 nuclei were counted. 


\section{Results}

PREPARATION OF SAMPLES

Freshly collected preparations of amniotic cells, analysed after Giemsa staining or by phase contrast microscopy, showed that only a small proportion $(5-15 \%)$ of nuclei were intact and free of cytoplasm. Our past results have shown that cells surrounded by cytoplasm result in poor hybridisation signals after FISH and are not ideal for FISH tests.

ANALYSIS OF METAPHASE SPREADS

The frequency of correct, specific hybridisation signals obtained using the various chromosome 21 derived probes (table 1) was initially evaluated on metaphase preparations from peripheral blood lymphocytes. Using the $\alpha$ satellite probe L1.26, hybridisation complexes on the centromeric region of both homologous chromosomes 13 and 21 could be detected in about $90 \%(n=20)$ of metaphases prepared from nine normal persons. In four trisomy 21 patients five hybridisation complexes could be seen. However, using this probe, the intensity of the signal varied greatly from one sample to another; in about one third of normal subjects the centromeric hybridisation complex on one of the two chromosomes 21 was very small.

With cosmid D12118 (ICRFc102D12118) and YAC yGART2, mapping to 21q22.3 and 21 q22.1 respectively, over $90 \% \quad(n=20)$ of metaphase spreads from four normal subjects showed hybridisation signals on both chromosomes 21 .

Seventeen cosmids obtained from a chromosome 21 library were each tested by FISH on metaphase spreads from normal lymphocytes in order to establish their specificity and to define the conditions required for their use as probes for FISH. All cosmids were confirmed as mapping to the $21 \mathrm{q} 22.3$ region. The cosmid contig $242 \mathrm{c}$ gave clear hybridisation signals on each of the two chromosomes 21 on about $95 \%(n=20)$ of metaphase spreads of a lymphocyte preparation from a normal subject. When contig 242c, labelled with digoxigenin and visualised with TRITC was used in conjunction with contig cCMP21.a, labelled with biotin and visualised with fluorescein, doublet signals (one red and one green) could be observed on the long arm (21q22.3 and proximal 21q22) of both homologous chromosomes 21 in about $90 \%(n=20)$ of metaphase spreads.
FISH TESTS ON LYMPHOCYTES IN INTERPHASE

The $\alpha$ satellite L1.26 probe showed great variability in hybridisation signals. The expected four hybridisation complexes, with the centromeres of chromosomes 21 and 13, could be observed in 38 to $69 \%(n=100)$ of nuclei prepared from normal lymphocytes. A further 15 to $25 \%$ of nuclei showed three signals reflecting the observed weak reaction on one of the homologous chromosomes 21 owing to polymorphic variation or the coalescence of two hybridisation complexes because of nucleolar association of chromosomes 21 and 13.

The L1.26 probe was tested on lymphocytic nuclei from three patients with trisomy 21 ; in two patients only $42 \%$ and $51 \%(n=100$ in each case) of nuclei showed the expected five signals. However, in a mosaic patient $(46, \mathrm{XY})$ $47, \mathrm{XY},+21)$ a good correlation was observed between the percentage of metaphase spreads with three chromosomes 21 reacting with the probe $(62 \%, n=20)$ and the percentage of nuclei in interphase with five hybridisation complexes $(58 \%, \mathrm{n}=100)$.

Cosmid D12118 was tested simultaneously on two preparations of lymphocyte nuclei from a normal person and a patient with trisomy 21 . If nuclei showing no hybridisation signals were excluded, the expected two and three signals were seen in $78 \%(n=125)$ and $43 \%(n=79)$ of nuclei respectively.

The YAC probe yGART2, used to evaluate two normal preparations resulted in the presence of two signals in $60 \%(n=40$ and $n=47)$ of hybridised nuclei. These signals were similar in size to those observed with the single cosmid although the probe is about 15 times larger than the cosmid.

The cosmid contig 242c, tested on four samples, produced two clear signals in an average of $81 \%(\min =71, \max =86)$ of hybridised nuclei (mean number of hybridised nuclei counted $=79, \min =35, \max =101$ )

FISH TESTS ON AMNIOTIC CELLS IN INTERPHASE

Only about 5 to $15 \%$ of amniotic cells present on a single slide showed hybridisation complexes. No improvement was seen when protease and paraformaldehyde treatments were included in the protocol. Results on those nuclei showing at least one signal ("hybridised nuclei") are summarised in table 2 .

The $\alpha$ satellite probe $\mathrm{L} 1.26$ was repeatedly

Table 2 Results of FISH tests on amniotic cells in interphase

\begin{tabular}{|c|c|c|c|c|c|c|}
\hline Probe & Sample details* & No of samples & $\begin{array}{l}\text { No of samples } \\
\text { analysed }\end{array}$ & $\begin{array}{l}\text { No of hybridised nuclei } \\
\text { counted. } \dagger \text { Data shown as } \\
\text { mean (min, max) }\end{array}$ & $\begin{array}{l}\text { \% hybridised nuclei with } \\
\text { the expected number of } \\
\text { signals. } \ddagger \text { Data shown as } \\
\text { mean (min, max) }\end{array}$ & $\begin{array}{l}\text { \% hybridised nuclei in a } \\
\text { normal sample showing } \\
\text { three signals. Data shown } \\
\text { as mean (min, max) }\end{array}$ \\
\hline $\begin{array}{l}\text { D12118 } \\
\text { yGART2 } \\
242 c \\
242 c+ \\
\text { cCMP21.a }\end{array}$ & $\begin{array}{l}N \mathrm{C} \\
\mathrm{T} C \\
\mathrm{~N} C \\
\mathrm{~T} \mathrm{C} \\
\mathrm{N} \mathrm{U} \\
\mathrm{T} \mathrm{U} \\
\mathrm{N} \mathrm{U} \\
\mathrm{T} \mathrm{U}\end{array}$ & $\begin{array}{r}27 \\
1 \\
7 \\
2 \\
50 \\
4 \\
29 \\
5\end{array}$ & $\begin{array}{r}22 \\
1 \\
7 \\
2 \\
22 \\
2 \\
28 \\
5\end{array}$ & $\begin{array}{c}50(14,197) \\
83 \\
54(10,161) \\
175(72,277) \\
23(10,50) \\
40(14,66) \\
42(16,68) \\
37(10,100)\end{array}$ & $\begin{array}{l}50(26,70) \\
33 \\
47(25,65) \\
44(40,47) \\
72(41,100) \\
56(50,61) \\
74(50,87) \\
54(49,88)\end{array}$ & $\begin{array}{l}14(0,44) \\
\text { N/A } \\
14(0,33) \\
\text { N/A } \\
7(0,31) \\
\text { N/A } \\
1(0,4) \\
\text { N/A }\end{array}$ \\
\hline
\end{tabular}

* Samples were obtained from normal $(\mathrm{N})$ or trisomic $(\mathrm{T})$ patients. Some were cultured $(\mathrm{C})$ before being used for FISH analysis; others were not $(\mathrm{U}=$ uncultured) t Only hybridised nuclei, that is, those showing one or more hybridisation signal, were analysed.

$\ddagger$ L1.26 was expected to show four hybridisation signals on normal samples and five on samples from patients with trisomy 21 . D12118, yGART2, and 242c were expected to show two and three signals on normal and trisomic samples respectively. 242c and cCMP21.a, differentially labelled and used simultaneously, were expected to show two and three doublet signal on normal and trisomic samples respectively. 
tested without any success: the signals tended to coalesce and it was extremely difficult to assess their number in each nucleus.

Cosmid D12118 was tested on nuclei from 27 normal amniotic samples that had been cultured. Five samples were uninformative owing to inefficient lysis and consequential lack of hybridisation. In the other 22 samples between 26 and $70 \%$ (mean number of nuclei counted $=50$ ) of nuclei showed two hybridisation complexes and 20 samples showed up to $44 \%$ of nuclei with three signals thus giving false positive results.

When tested on a trisomy 21 amniotic sample, $33 \%(n=83)$ of nuclei had three signals and another $35 \%$ had two signals; conventional cytogenetic analysis showed no evidence that this patient was a mosaic.

The YAC probe yGART2 was used to test seven cultured normal amniocyte samples; 33 to $65 \%$ (mean number of hybridised nuclei $=54$, $\min =10, \max =161$ ) of hybridised nuclei displayed the expected two signals and in five of the samples up to $33 \%$ of nuclei showed three (table 2). Two trisomic samples showed three signals in $40 \%(n=72)$ and $47 \%(n=277)$ of hybridised nuclei.

The reaction of the $242 \mathrm{c}$ cosmid contig was tested on 50 uncultured amniocyte samples from normal pregnancies. Twenty-eight of these samples $(56 \%)$ contained too few nuclei $(<10)$ to be analysed. In the remaining 22 samples, the mean number of hybridised nuclei was $23(\min =10, \max =50) ; 14$ samples showed two clear signals in $70 \%$ to $100 \%$ of nuclei and eight showed the expected two hybridisation complexes in 41 to $62 \%$. However, in 15 samples up to $31 \%$ of the normal nuclei displayed three signals (fig 1, table 2).

Of four amniotic samples from trisomy 21 fetuses, two showed too few hybridised nuclei $(<10)$ to be analysed. The other two showed the expected three signals in over $50 \%$ of the nuclei that could be analysed (fig 1 , table 2 ).

The two cosmid contigs 242c and cCMP21.a (labelled with digoxigenin and biotin respectively) were used simultaneously to evaluate 29 uncultured amniotic samples from normal fetuses. Only doublets composed of one red and

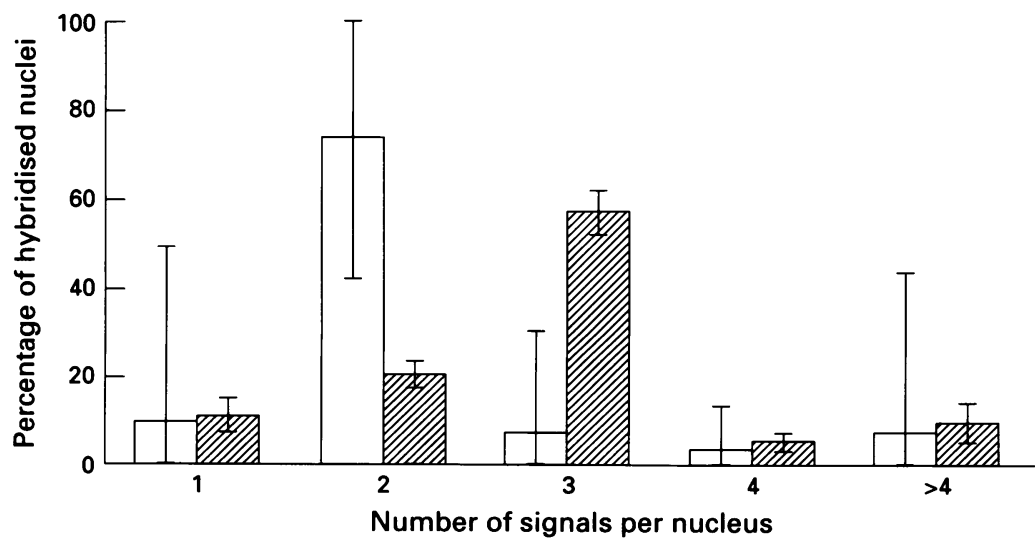

Figure 1 Probe 242c was used to evaluate 22 uncultured amniotic samples from normal fetuses (unshaded) and two from trisomic fetuses (shaded). The proportion of nuclei showing one, two, three, four, or more than four signals were calculated as a percentage of hybridised nuclei. Bars indicate the maximum and minimum percentages observed. one green signal were counted (fig 2). One sample contained too few nuclei for analysis $(<10)$. Of the other 28 samples tested, three showed 16 to 20 hybridised nuclei per slide and the remainder showed 50 or more; all 28 samples displayed two doublet signals in 50 to $87 \%$ of hybridised nuclei (table 2 ). The most prominent feature of the distribution data was the low proportion (nine out of 28) of these normal samples showing artefactual third signals; in none of these nine samples was the frequency of three signals greater than $5 \%$ (table 2, fig 3).

When this double labelling approach was used to evaluate five trisomic samples, three doublet signals were seen in $49 \%$ to $88 \%$ of hybridised nuclei (mean number of hybridised nuclei $=37, \min =10, \max =100$ ) (fig 3 ).

\section{Discussion}

The main technical difficulties in using FISH on uncultured amniocytes for prenatal diagnostic tests derive from the characteristics of these cells. Amniotic fluid samples contain a heterogeneous population of cells; only a few are viable and will grow in vitro. ${ }^{25-27}$ Probably some cells, like the squamous cells present in buccal smears, are resistant to lysis and there-
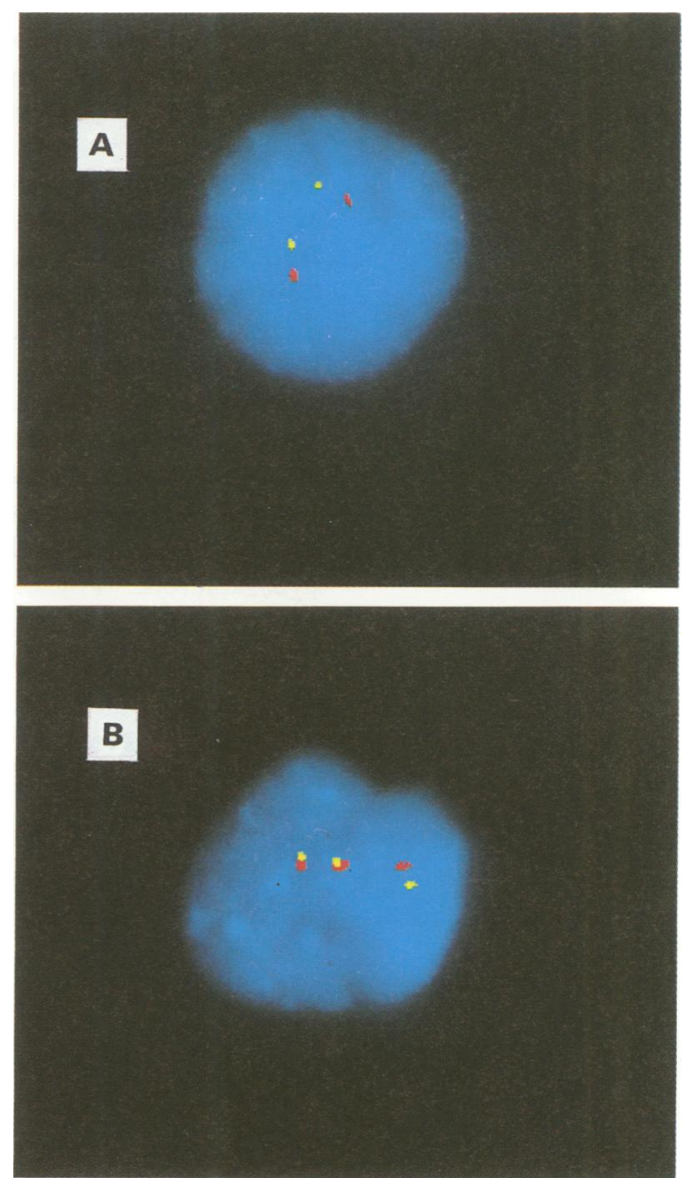

Figure 2 Detection of chromosome 21 aneuploidy in uncultured amniocytes samples using two differentially labelled cosmid contigs: $242 c$ and cCMP21.a (detected labelled cosmid contigs: $242 c$ and $c C M P 21 . a$ (detected
with TRITC and FITC respectively). Two doublet signals were seen in the nuclei of a normal sample $(A)$ whereas three were seen in a sample from a Down's syndrome fetus $(B)$. 


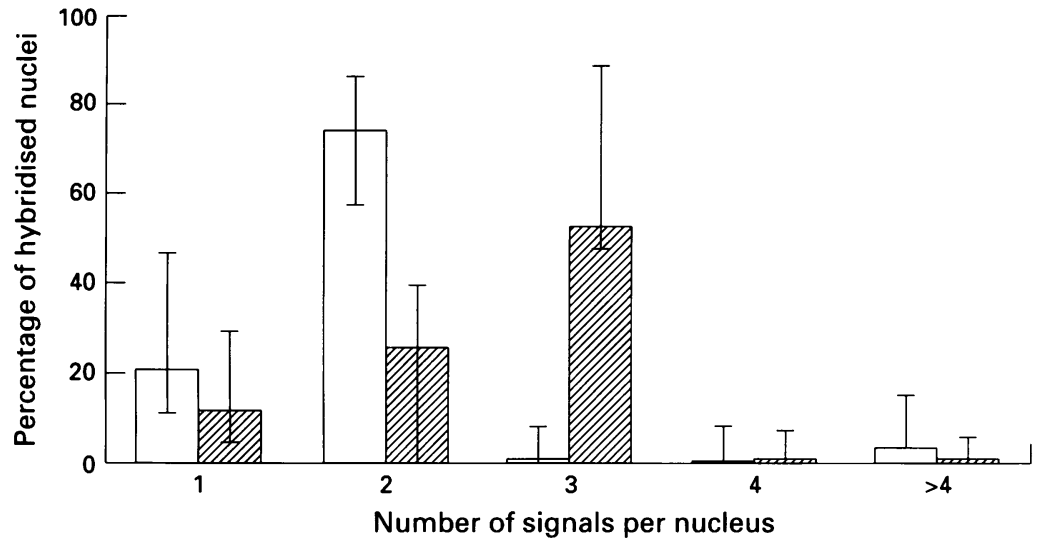

Figure 3 Probes 242c and cCMP21.a were used to evaluate 28 uncultured amniotic samples from normal fetuses (unshaded) and five from trisomic samples (shaded). Figures were calculated as a percentage of hybridised nuclei. Bars indicate the maximum and minimum percentages observed.

fore unsuitable for FISH tests. ${ }^{28}$ It is also plausible that the large proportion of dead cells present in amniotic fluid is resistant to lysis. In our investigation (in agreement with previous studies $^{12-14}$ ) only a small proportion of nuclei (approximately 5 to $15 \%$ ) was suitable for FISH analysis.

While all 21 derived probes analysed provided clear, specific signals in over $90 \%$ of metaphase spreads from peripheral blood lymphocytes, the frequency of correct signals was lower when these probes were tested on nuclei in interphase. Two signals were observed in 71 to $85 \%$ of lymphocytes from normal subjects analysed using cosmid D12118 and contig 242c and three signals were observed in up to $43 \%$. When trisomy 21 samples were investigated the frequency of the expected three signals varied between $43 \%$ and $60 \%$; therefore there was not a clear discrimination between normal and trisomic samples.

The observation of false positives, that is, spurious third signals in nuclei from normal samples, was attributed to hybridisation of the probe to low affinity sites or non-specific binding of fluorochrome labelled ligand to the cell cytoskeleton. These artefactual signals were largely distinguished and discounted by the simultaneous use of two differentially labelled cosmid contigs. Only doublets composed of one red and one green spot were accepted as true signals on the basis that the non-specific sites would be unlikely to be labelled by both contigs (fig 2). This dramatically decreased the percentage of normal nuclei showing three signals and thus improved the distinction between normal and trisomic samples (table 2, fig 3) and therefore the diagnostic value of the test. Another improvement attributed to the simultaneous use of the two probes was the increased confidence with which signals could be counted in the presence of high levels of fluorescent background. This presumably explains the lower proportion of samples with too few hybridised nuclei for analysis (only one out of 29) compared with that observed when the single cosmid contig was used ( 28 out of 50 ); weak or unclear signals which had been previously discounted when using a single cosmid contig were accepted as true signals when seen as differentially labelled doublets.

The observation that the percentage of nuclei from a trisomic patient showing three signals was always lower than that of a normal patient showing two signals is expected; if the probability of a probe to hybridise with one chromosome is 0.8 , the probability of seeing two signals in normal nuclei is $0 \cdot 8^{2}$, that is $0 \cdot 64$. In trisomic patients the percentage of nuclei showing three hybridisation signals should be $0 \cdot 8^{3}$, that is 0.51 .

Upon statistical analysis of the results obtained for normal samples using both probes simultaneously, the probability of a particular normal cell having exactly three signals is approximately 0.01 . Therefore the probability of allocating trisomic samples as normal is very low. When the two contigs were used simultaneously to evaluate trisomic samples, $54 \%$ of the total nuclei counted had three signals; therefore the probability of trisomic samples being classified as normal is also extremely low (less than 0.001 if 15 nuclei are counted).

Larger prospective trials are needed to establish the clinical reliability of the double labelling technique for the detection of trisomy 21 on uncultured amniocytes. The use of 5 to $10 \mathrm{ml}$ of amniotic fluid as opposed to less than $2 \mathrm{ml}$ would result in a larger proportion of samples containing adequate numbers of nuclei for analysis. By considerably reducing the likelihood of scoring adventitious signals the double labelling technique does appear to improve greatly the discrimination of normal from trisomic cells in interphase FISH analysis.

We thank the South East Thames Regional Genetics Centre for the provision of samples, Andreas Gnirke, Yun Ling Zheng, and Nigel Carter for the provision of some probes used in this study, Nigel Smeeton and Paul Scriven for the statistical analysis, and Nigel Smeeton and Paul Scriven for the statistical analysis, and Adrienne Knight for her assistance in the preparation of the manuscript. This work was supported by grants from Action Research, The Generation Trust, and the Medical Research
Council.

1 Schröder J. Transplacental passage of blood cells. $\mathcal{f}$ Med Genet 1975;12:230-42.

2 Adinolfi $M$. On a non-invasive approach to prenatal diagnosis based on the detection of fetal nucleated cells in maternal blood samples. Prenat Diagn 1991;11:799-804.

3 Rhine SA, Palmer CG, Thompson JF. A simple alternative to amniocentesis for first trimester prenatal diagnosis. Birth Defects 1977;12:231-47.

4 Adinolfi M, Davies A, Sharif S, Soothill P, Rodeck C Detection of trisomy 18 and $Y$-derived sequences in fetal nucleated cells obtained by transcervical flushing. Lancet nucleated cells

5 Devilee $\mathrm{P}$, Cremer T, Slagboom $\mathrm{P}$, et al. Two subsets of human alphoid repetitive DNA show distinct preferential human alphoid repetitive DNA show distinct preferential 18 and 21. Cytogenet Cell Genet 1986;41:193-201.

6 Bartsch O, Schwinger E. A simplified protocol for fluoresBartsch O, Schwinger E. A simplified protocol for fluores-
cence in situ hybridisation with repetitive DNA probes and its use in clinical cytogenetics. Clin Genet 1991;40:47-56.

7 Weier H-U, Gray JW. A degenerate alpha satellite probe, detecting a centromeric deletion on chromosome 21 in an apparently normal human male, shows limitations of the use of satellite DNA probes for interphase ploidy analysis. Anal Cell Pathol 1992;4:81-6.

8 Julien C, Bazin A, Guyot B, Forestier F, Daffos F. Rapid prenatal diagnosis of Down's syndrome with in situ hybridisation of fluorescent probes. Lancet 1986;ii:863-4.

Lichter P, Cremer T, Tang CJC, Watkins PC, Manuelidis L Ward DC. Rapid detection of human chromosome 2 aberrations by in-situ hybridization. Proc Natl Acad Sci USA 1988;85:9664-8.

10 Pinkel D, Landegent J, Collins C, et al. Fluorescence in-situ hybridization with human chromosome-specific libraries: detection of trisomy 21 and translocations of chromosome 4. Proc Natl Acad Sci USA 1988;85:9138-42.

11 Kuo WL, Tenjin H, Segraves R, Pinkel D, Golbus MS, Gray J. Detection of aneuploidy involving chromosomes 13,18 or 21 by fluoresence in-situ hybridization (FISH) to interphase and metaphase amniocytes. Am $\mathcal{J}$ Hum Genet 1991;49:112-9. 
12 Zheng YL, Ferguson-Smith MA, Warner JP, FergusonSmith ME, Sargent CA, Carter NP. Analysis of chromosome 21 copy number in uncultured amniocytes by fluorescence in-situ hybridization using a cosmid contig. Prenat Diagn 1992;12:931-43.

13 Klinger K, Landes G, Shook D, et al. Rapid detection of chromosome aneuploidies in uncultured amniocytes by using fluorescence in situ hybridisation (FISH). Am $\mathcal{F ~ H u m}$ Genet 1992;51:55-65.

14 Ried T, Landes G, Dackowski W, Klinger K, Ward DC. Multicolor fluorescence in-situ hybridization for the simultaneous detection of probe sets for chromosomes 13, 18,21, $\mathrm{X} \& \mathrm{Y}$ in uncultured amniotic fluid cells. Hum Molec Genet 1992;1:307-13.

15 Ward BE, Gersen SL, Carelli MT, et al. Rapid prenatal diagnosis of chromosomal aneuploidies by fluorescence in situ hybridisation: clinical experience with 4,500 specisitu hybridisation: clinical experience

16 Rooney DE, Czepulkowski BH. Prenatal diagnosis and tissue culture. In: Rooney DE, Czepulkowski BH, eds. Human cytogenetics: a practical approach. 2nd ed. Oxford: IRL Press, 1992:55-89.

17 Gosden CM, Davidson C, Robertson M. Lymphocyte culture. In: Rooney DE, Czepulkowski BH, eds. Human cytogenetics: a practical approach. 2nd ed. Oxford: IRL Press, 1992:31-54.

18 Sambrook J, Fritsch EF, Maniatis T. Molecular cloning: a laboratory manual. New York: Cold Spring Harbor Laboratory Press, 1989.

19 Gnirke A, Barnes TS, Patterson D, Schild D, Featherstone $T$, Olson MV. Cloning and in vivo expression of the T, On GART gene using yeast artificial chromosomes. EMBO f 1991;10:1629-34.
20 Anand R, Villasante A, Tyler-Smith $\mathrm{C}$. Construction of yeast artificial chromosome libraries with large inserts using fractionation by pulsed-field gel electrophoresis. Nucleic Acids Res 1989;17:3425-33.

21 Southern EM, Anand R, Brown WRA, Fletcher DS. A model for the separation of large DNA molecules by crossed field gel electrophoresis. Nucleic Acids Res 1987;15:5925-43.

22 Lehrach H. In: Davies KE, Tilghman SM, eds. Genome analysis. Vol 1. Genetic and Physical Mapping. New York: Cold Spring Harbor Laboratory Press, 1990:39-81.

23 Knott V, Rees DJD, Cheng Z, Brownlee GG. Randomly picked cosmid clones overlap the pyrB and the oriC gap in the physical map of the $E$ coli chromosome. Nucleic Acids Res 1988;16:2601-12.

24 Holland J, Coffey AJ, Gianelli F, Bentley DR. Vertical integration of cosmid and YAC resources for interval integration of cosmid and YAC resources for interval map.

25 Martin AO. Characteristics of amniotic fluid cells in vitro and attempts to improve culture techniques. Clin Obstet Gynaecol 1980;7:143-66.

26 Harris A. Glycoproteins that distinguish different cell types found in amniotic flud. Hum Genet 1982;62:188-92.

27 Hoehn H. Amniotic fluid cell culture. In: Milunsky A, ed. Genetic disorders and the fetus: diagnosis, prevention and treatment. 3rd ed. Baltimore: John Hopkins University Press, 1992.

28 Burns J, Chan VTW, Jonasson JA, Fleming KA, Taylor S, McGee JO'D. Sensitive system for visualising biotinylated DNA probes hybridised in situ: rapid sex determination of intact cells. $\mathcal{f}$ Clin Pathol 1985;38:1085-92. 